\title{
OPTIMIZATION OF TUNED MASS DAMPERS ATTACHED TO DAMPED STRUCTURES - MINIMIZATION OF MAXIMUM DISPLACEMENT AND ACCELERATION
}

\author{
JAN ŠTĚPÁNEK*， JiŘí MÁCA \\ Czech Technical University in Prague, Faculty of Civil Engineering, Department of Mechanics, Thákurova 7, \\ 16629 Prague 6, Czech Republic \\ * corresponding author: jan.stepanek@fsv.cvut.cz
}

\begin{abstract}
A tuned mass damper is a device, which can be highly helpful while dealing with dynamic behaviour of structures. Its proper design is conditioned by knowledge of both loading and the structure properties. In many cases, the structure can be represented by single degree of freedom model, which simplifies the design and optimization of tuned mass dampers. Most of studies focus only on minimization of displacement of the main structure under harmonic force load, however, in many cases, different frequency response function would be more appropriate. This paper presents an extension of design formulas for the $H_{\infty}$ optimization of tuned mass dampers for damped structures and various frequency response functions.
\end{abstract}

KEYWORDS: Acceleration, damped structure, displacement, dynamic response, optimization, tuned mass damper.

\section{Introduction}

Optimal parameters of a tuned mass damper (TMD) have been a subject of many researches since the first half of the $20^{\text {th }}$ century. Since then, several different optimization criteria have been proposed. The most common ones are called $\mathrm{H}_{2}$, which minimizes the area under a frequency response function (FRF) and $H_{\infty}$, which minimizes the maximum amplitude magnification factor. A first set of nearly optimal TMD parameters for undamped structure came from the existence of invariant points, where the amplitude is independent of TMD damping $[1,2]$.

A closed form solution of $H_{\infty}$ optimum parameters of TMD attached to a damped structure have not been found. In 1978, Ioi and Ikeda presented empiric design formulas [3], however, they were derived only for an insufficiently large mass ratio $\mu>0.03$, which makes them inappropriate for certain civil engineering structures. It is interesting that despite the formulas being derived for a large mass ratio, they are recommended in footbridge design guidebook SETRA [4]. Design charts allowing use of lower mass ratio $\mu>0.01$ were published in 1981 [5]. Asami et al. used a perturbation method to modify the fixed-point solution regarding a main system damping ratio as a perturbation [6]. They obtained a series solution, which is, however, too complicated for engineering application. Therefore, they have proposed a simplified version. Abubakar presented a simple modification of the fixed-point TMD optimum for damped structure. This modification provides very accurate solution for minimization of displacement [7]. Despite many useful formulas and design methods being proposed, some modern guidebooks for civil engineering, for example $[4,8]$, keeps using the original approximate solutions thanks to their easy application.

Most of studies focus on a reduction in a maximum amplitude of displacement. This is a suitable criterion for the reduction of structural stress due to a dynamic load. However, in many cases, the main purpose of TMD is to reduce the maximum amplitude of acceleration of the main structure. These cases contain machines and sensors which are sensitive to vibration, because the vibration tolerance is usually defined in the units of acceleration. Furthermore, human comfort criteria inside buildings or on footbridges are usually limited by acceleration.

This paper points out the importance of distinction between displacement and acceleration criteria of the $H_{\infty}$ optimization. The approximate optimal stiffness and damping ratio for both criteria are proposed in this paper covering both ground and force excitation. Considering the importance of easy practical application of the result, the formulas for optimal parameters are composed of standard solutions given by the fixed-point method and a correction, which takes nonzero damping into account. Therefore, in the case of zero structural damping, we obtain standard result given by the fixed-point method. In the case of nonzero damping, we obtain results given by the modified formulas.

\section{Equations of Motion}

The main structure is represented by a single degree of freedom (SDOF) system and it consists of a mass $m_{1}$, stiffness $k_{1}$, and damping $c_{1}$. TMD is composed of a mass $m_{2}$, an elastic member with stiffness $k_{2}$ and a viscous member with damping $c_{2}$. The struc- 


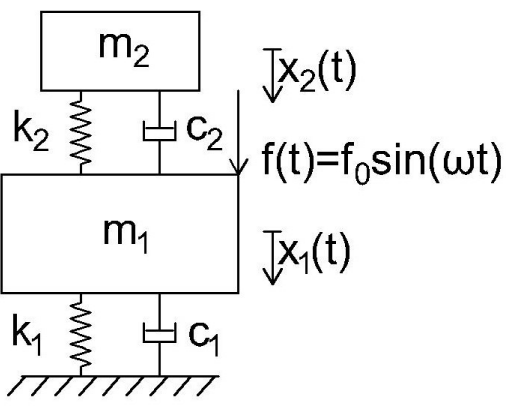

a)

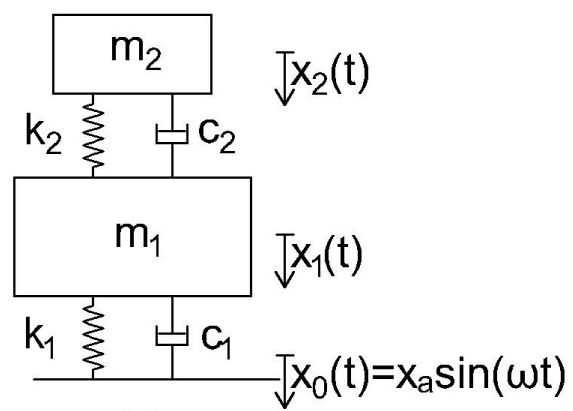

b)

FiguRE 1. a) Force excitation, b) Support excitation.

$$
\left[\begin{array}{cc}
k_{1}+k_{2} & -k_{2} \\
-k_{2} & k_{2}
\end{array}\right]\left\{\begin{array}{l}
x_{1} \\
x_{2}
\end{array}\right\}+\left[\begin{array}{cc}
c_{1}+c_{2} & -c_{2} \\
-c_{2} & c_{2}
\end{array}\right]\left\{\begin{array}{l}
\dot{x_{1}} \\
\dot{x_{2}}
\end{array}\right\}+\left[\begin{array}{cc}
m_{1} & 0 \\
0 & m_{2}
\end{array}\right]\left\{\begin{array}{l}
\ddot{x_{1}} \\
\ddot{x_{2}}
\end{array}\right\}=F(t)
$$

ture can be loaded by a harmonic force (Figure 1a) or excited by support motion (Figure 1b). This paper covers both loading cases, because the optimal parameters of TMD are not identical for force and support excitation. The equations of motion are described by Eq. 1, where $F(t)=\left\{\begin{array}{c}f(t) \\ 0\end{array}\right\}$ in the case of force excitation, and $F(t)=\left\{\begin{array}{l}-\ddot{x}_{0}(t) m_{1} \\ -\ddot{x_{0}}(t) m_{2}\end{array}\right\}$ in the case of support excitation. $x, \dot{x}$ and $\ddot{x}$ denote the displacement, velocity and acceleration, respectively. It is important to note that $x$ induced by support excitation is a relative displacement between the support and the mass. The absolute displacement is $x^{a}=x+x_{0}$.

\section{Optimal PARAMETERS}

In order to solve Equation 1, it is advantageous to use following dimensionless substitutions and notation for further operations [9]:

$\omega_{1}=\sqrt{k_{1} / m_{1}} \quad$ natural frequency of the main $\omega_{2}=\sqrt{k_{2} / m_{2}} \quad \begin{aligned} & \text { structure, } \\ & \text { natural frequency of TMD, }\end{aligned}$

$\mu=m_{2} / m_{1} \quad$ mass ratio,

$\beta=\omega_{2} / \omega_{1} \quad$ frequency ratio,

$\Omega=\omega / \omega_{1}$

$c_{1, c r}=2 \sqrt{k_{1} m_{1}}$

forcing frequency ratio,

critical damping of the main structure,

$c_{2, c r}=2 \sqrt{k_{2} m_{2}} \quad$ critical damping of TMD,

$\xi_{1}=c_{1} / c_{1, c r} \quad$ damping ratio of the main structure,

$\xi_{2}=c_{2} / c_{2, c r} \quad$ damping ratio of TMD,

$N_{f}(\Omega)=\left(2 \xi_{2} \beta \Omega\right)^{2}+\left(\beta^{2}-\Omega^{2}\right)^{2}$,

$N_{a}(\Omega)=\left[1+\left(2 \xi_{1} \Omega\right)^{2}\right]\left[\left(\beta^{2}-\Omega^{2}\right)^{2}+\left(2 \xi_{2} \beta \Omega\right)^{2}\right]$,

$N_{r}(\Omega)=\left[(1+\mu) \beta^{2}-\Omega^{2}\right]^{2} \Omega^{4}+(1+\mu)^{2}\left(2 \xi_{2} \beta \Omega\right)^{2} \Omega^{4}$,

$D(\Omega)=\left[\left(1-\Omega^{2}\right)\left(\beta^{2}-\Omega^{2}\right)-\mu \beta^{2} \Omega^{2}-4 \xi_{1} \xi_{2} \beta \Omega^{2}\right]^{2}+$ $+4 \Omega^{2}\left[\left(\beta^{2}-\Omega^{2}\right) \xi_{1}+\left(1-\Omega^{2}-\mu \Omega^{2}\right) \beta \xi_{2}\right]^{2}$.

Assuming a harmonic load according to Figure 1, a steady state part of response can be expressed from
Eq. 1. The frequency response functions which describe the normalized response of the structure are summarized in Table 1.

\subsection{FIXED-POINT APPROACH}

The approximate $H_{\infty}$ optimum for main structure with no or negligible damping can be reached by the fixed-point method. This method was firstly described by Den Hartog [1]. He postulated that the approximate optimum frequency ratio $\beta_{o p t}^{u}$ is reached if the value of FRF is equal in invariant points $P$ and $Q$, where the value of FRF is not affected by TMD damping $c_{2}$. Brock lately stated that the optimum damping ratio $\xi_{2, \text { opt }}^{u}$ can be taken as the average of two optima, each calculated separately for one of two points $P$ and $Q$ and he derived the well known formula of optimum damping for FRF No. 1 in Table 1 [2]. Using this approach, we can derive the approximate optimum solutions for various FRFs. The results are summarized in Table1. It should be noted that the optimum parameters are identical for transfer functions 1 and 3 , as well as for 2 and 4 . This is can be seen in $N_{a}\left(\Omega, \xi_{1}=0\right)=N_{f}(\Omega)$. However, this is true only if $\xi_{1}=0$.

\subsection{NON-ZERO DAMPING}

The fixed-point approach is not available if we introduce damping of the main structure. An analytical solution of optimum TMD parameters attached to a damped main structure have not been found and according to Asami et al., the solution is probably impossible [6]. Therefore, we need to rely on empiric and approximate solutions.

In order to define an empiric formulas for optimum frequency ratio $\beta_{\text {opt }}$ and optimum damping ratio $\xi_{\text {opt }, 2}$, the values of the parameters were found numerically on a surface of $44 \times 41$ points for $0.001 \leq \mu \leq 0.2$ and $0 \leq \xi_{1} \leq 0.2$. The density of mesh was increased in the area of low mass ratio $0.001 \leq \mu \leq 0.005$, because in this range, the optimum values of $\beta_{\text {opt }}$ and 


\begin{tabular}{|c|c|c|c|c|c|c|}
\hline No. & excitation & $\begin{array}{l}\text { frequency response } \\
\text { function type }\end{array}$ & $\begin{array}{r}\text { freque } \\
\mathbf{f}\end{array}$ & $\begin{array}{l}\text { ncy response } \\
\text { function }\end{array}$ & $\beta_{o p t}^{u}$ & $\xi_{2, o p t}^{u}$ \\
\hline 1 & force & displacement & $\mid \frac{x_{1}}{f_{0} / k_{1}}$ & $-\mid=\sqrt{\frac{N_{f}(\Omega)}{D(\Omega)}}$ & $\frac{1}{1+\mu}$ & $\sqrt{\frac{3 \mu}{8(1+\mu)}}$ \\
\hline 2 & force & acceleration & $\frac{\ddot{x}_{1}}{\omega_{1}^{2} f_{0} / k_{1}}$ & $-\mid=\Omega^{2} \sqrt{\frac{N_{f}(\Omega)}{D(\Omega)}}$ & $\sqrt{\frac{1}{1+\mu}}$ & $\sqrt{\frac{3 \mu}{4(2+\mu)}}$ \\
\hline 3 & support & abs. displacement & $\left|\frac{x_{1}^{a}}{x_{0}}\right|$ & $=\sqrt{\frac{N_{a}(\Omega)}{D(\Omega)}}$ & $\frac{1}{1+\mu}$ & $\sqrt{\frac{3 \mu}{8(1+\mu)}}$ \\
\hline 4 & support & abs. acceleration & $\left|\frac{{\ddot{x_{1}}}^{a}}{\omega_{1}^{2} x_{0}}\right|$ & $=\Omega^{2} \sqrt{\frac{N_{a}(\Omega)}{D(\Omega)}}$ & $\sqrt{\frac{1}{1+\mu}}$ & $\sqrt{\frac{3 \mu}{4(2+\mu)}}$ \\
\hline 5 & support & rel. displacement & $\left|\frac{\omega_{1}^{2} x_{1}}{\ddot{x}_{0}}\right|$ & $=\frac{1}{\Omega^{2}} \sqrt{\frac{N_{r}(\Omega)}{D(\Omega)}}$ & $\sqrt{\frac{2-\mu}{2(1+\mu)^{2}}}$ & $\sqrt{\frac{3 \mu}{4(1+\mu)(2-\mu)}}$ \\
\hline 6 & support & rel. acceleration & $\left|\frac{x_{1}}{x_{0}}\right|$ & $=\sqrt{\frac{N_{r}(\Omega)}{D(\Omega)}}$ & $\sqrt{\frac{2+\mu}{2(1+\mu)^{2}}}$ & $\sqrt{\frac{3 \mu}{8(1+\mu)}}$ \\
\hline
\end{tabular}

TABLE 1. Optimum parameters of TMD based on the fixed-point method.

$\xi_{\text {opt }, 2}$ change more rapidly then in the rest of the function. A convergence problem may occur while finding $\xi_{\text {opt }, 2}$ numerically, because a structural response is much more sensitive to TMD tuning than to its damping.

The empiric formulas are combination of the fixedpoint solution and a correction function, which takes the structural damping into account. The original approach [3] uses a correction function composed of 2nd order polynomial of $\mu$ and $\xi_{1}$, but we found it neither simplest nor best-fitting. Numerous types of functions, including polynomial, exponential, logarithmic and hyperbolic ones, were tested using a non-linear regression to find the best approximation of the optimum TMD setting regarding a simple application. Thanks to similar characteristics of the optimum parameters for all frequency response functions, two common function were defined:

$$
\begin{gathered}
\beta_{o p t}=\beta_{o p t}^{u}+\xi_{1}\left[a_{1} \xi_{1}+a_{2}\left(\frac{\mu}{1+\mu}\right)^{\frac{1}{3}}\right] \\
\xi_{2, o p t}=\xi_{2, o p t}^{u}+\xi_{1}\left[b_{1}+b_{2} \ln (\mu)+b_{3} \mu+b_{4} \mu \xi_{1}^{2}\right]
\end{gathered}
$$

As we can see in the shape of Eqs. (2) and (3), the optimum values of TMD parameters $\beta_{o p t}=\beta_{o p t}^{u}$ and $\xi_{2, o p t}=\xi_{2, o p t}^{u}$ if $\xi_{1}=0$. In other words, the formulas preserve the fixed-point approach if the main structure remains undamped.

The coefficient of determination was chosen as the comparative criterion for function fitting and it reached higher than 0.997 in all cases of FRFs for our selected function types. The maximum error of $\beta_{\text {opt }}$ was lower than $1 \%$. The maximum error of $\xi_{2, \text { opt }}$ reached up $5 \%$, but it occurred only for extremely small mass ratio, where the optimum damping is close to zero and such a high error may be produced rather by a precisions of the numerical solution than by an inaccurate approximation. The average relative error of $\xi_{2, \text { opt }}$ was lower than $1 \%$ for all transfer functions. The coefficients $a_{i}$ and $b_{i}$ were found using linear regression and they are summarized in Table 2. Figure 2 presents the improvement of FRF using desing formulas (2) and (3) for relative displacement of structure under support excitation.

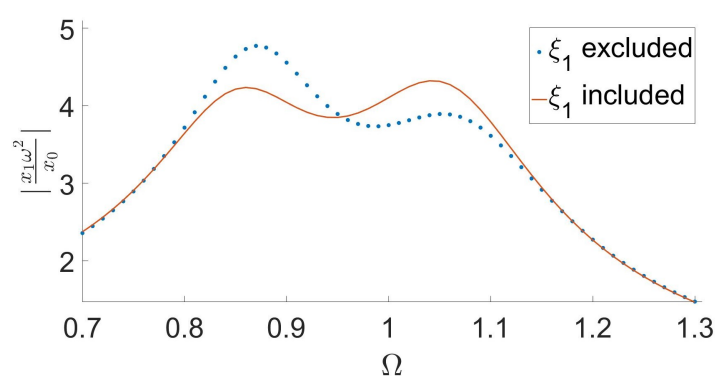

FiguRE 2. Improvement of TF $5\left(\mu=0.05, \xi_{1}=\right.$ $0.05)$ using desing formulas (2) and (3).

Figures 3-8 compare the numerical results with the approximations given by design formulas. We can see that in most cases, the empiric formulas give very precise results. It is also visible that $\xi_{2, o p t}$ rises as the structural damping increases. However, The effect of $\xi_{1}$ to the optimum frequency ratio is slightly more 


\begin{tabular}{ccccccc}
\hline FRF No. & $\boldsymbol{a}_{\mathbf{1}}$ & $\boldsymbol{a}_{\mathbf{2}}$ & $\boldsymbol{b}_{\mathbf{1}}$ & $\boldsymbol{b}_{\mathbf{2}}$ & $\boldsymbol{b}_{\mathbf{3}}$ & $\boldsymbol{b}_{\mathbf{4}}$ \\
\hline 1 & -0.7636 & -0.8748 & 0.1801 & 0.0140 & 0 & -4.6350 \\
2 & 1.2470 & 0.2644 & 0.2101 & 0.0178 & 1.264 & 0 \\
3 & -0.5561 & -0.8466 & 0.1782 & 0.01367 & 0.01865 & -4.3310 \\
4 & 1.5600 & 0.2778 & 0.1985 & 0.01552 & 1.1420 & 29.0900 \\
5 & -0.7639 & -1.5300 & 0.2005 & 0.01650 & 0.4629 & 0 \\
6 & 0.9731 & -0.2176 & 0.1783 & 0.0145 & 0 & 0 \\
\hline
\end{tabular}

TABle 2. Coefficients $a_{i}$ and $b_{i}$ for design equations (2) and (3).
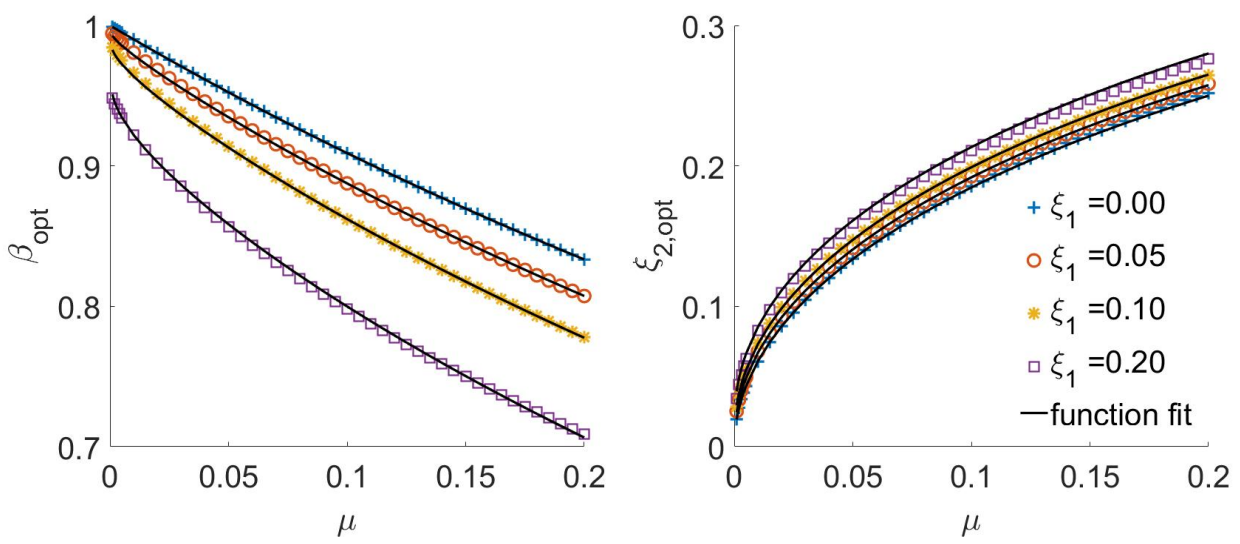

Figure 3. Optimum TMD parameters: TF No. 1.
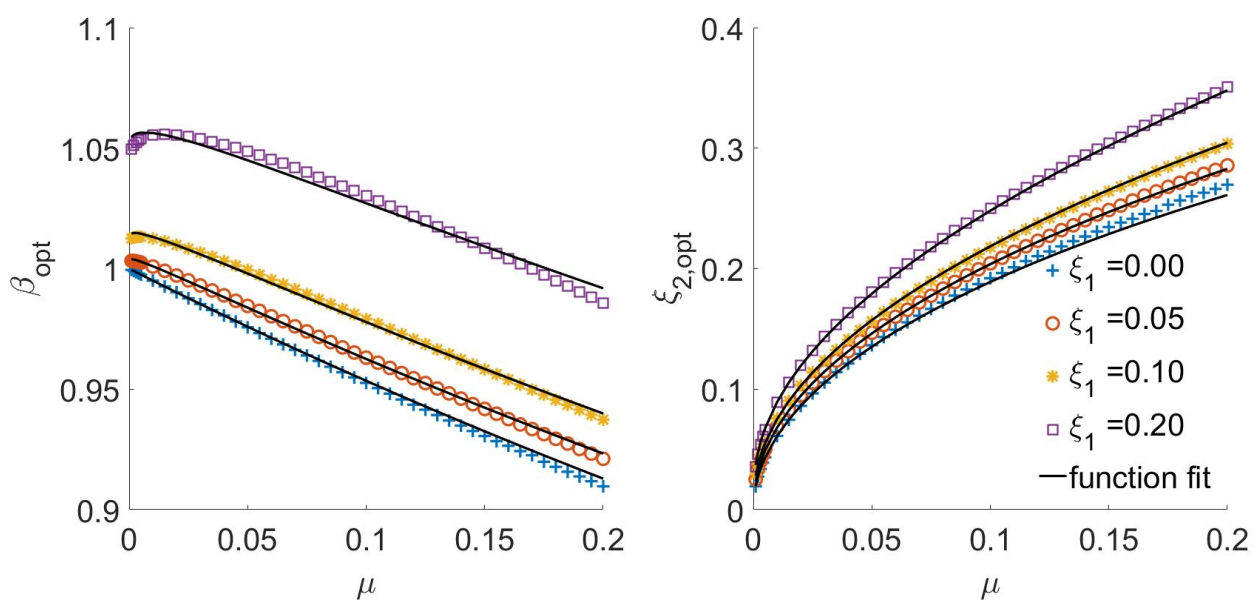

Figure 4. Optimum TMD parameters: TF No. 2.
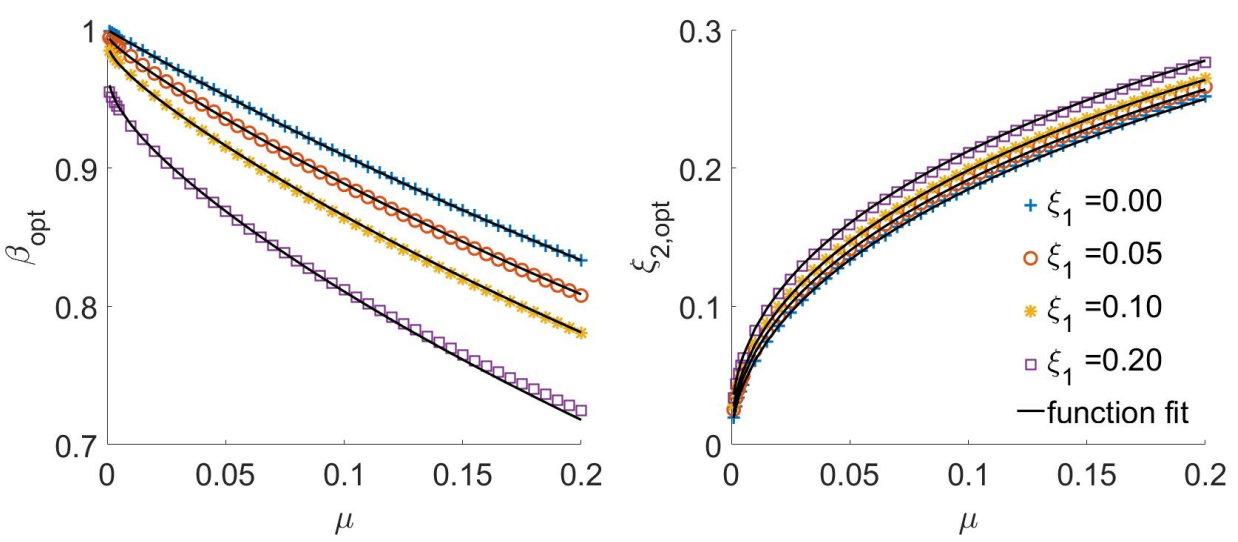

Figure 5. Optimum TMD parameters: TF No. 3. 

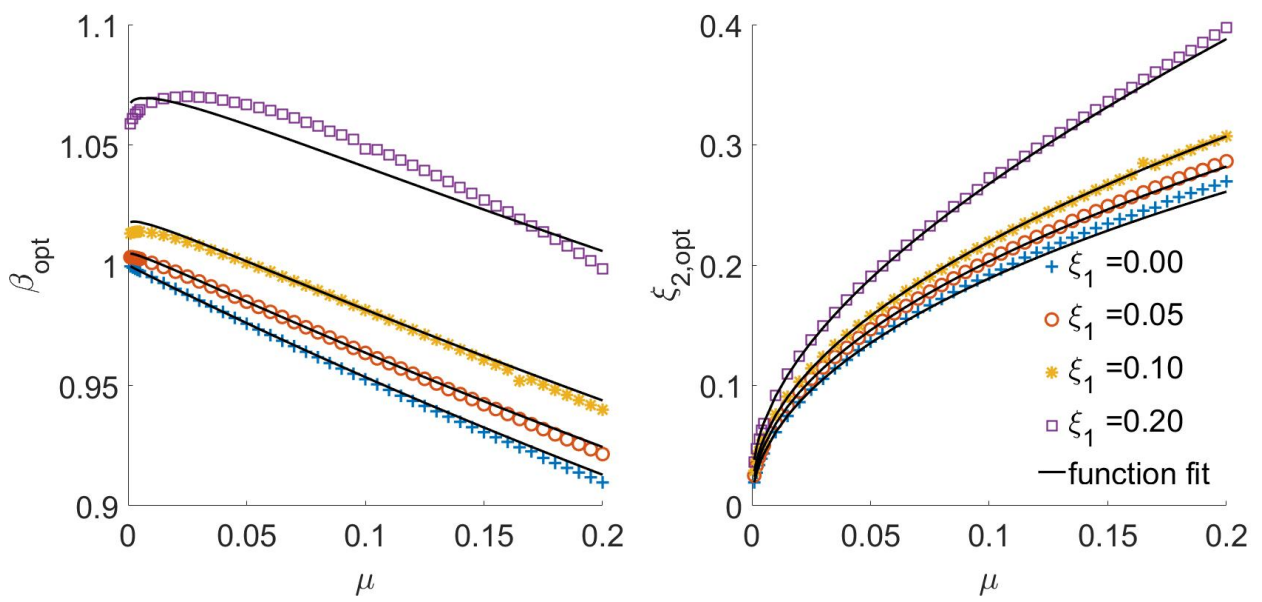

Figure 6. Optimum TMD parameters: TF No. 4.
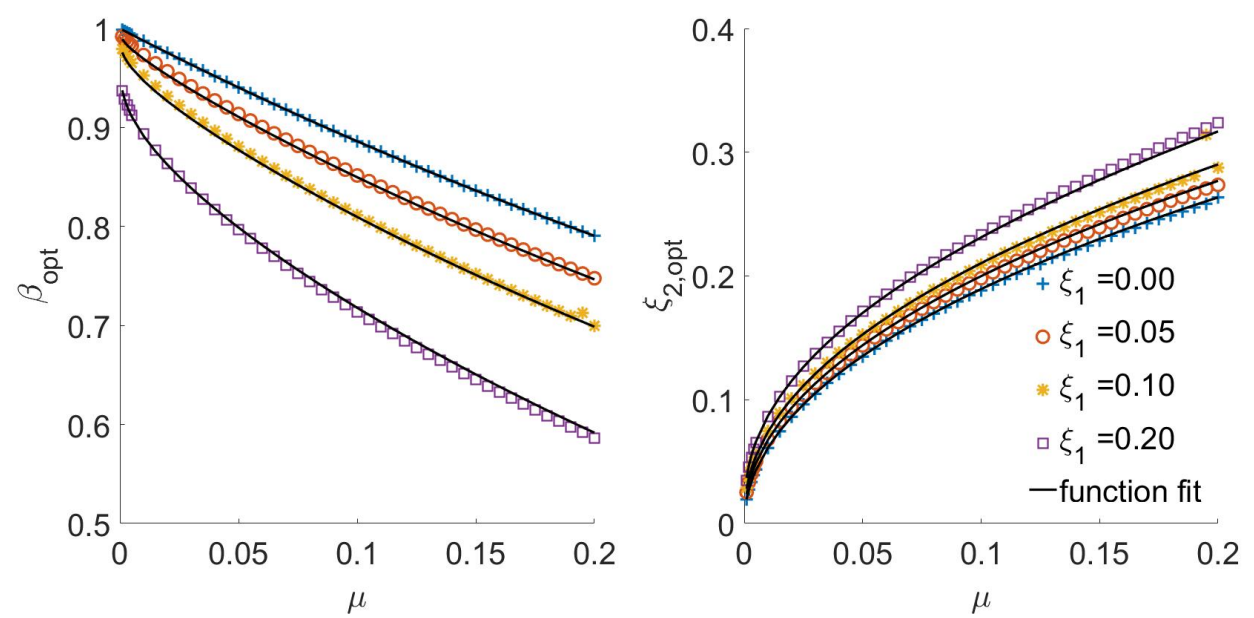

Figure 7. Optimum TMD parameters: TF No. 5.
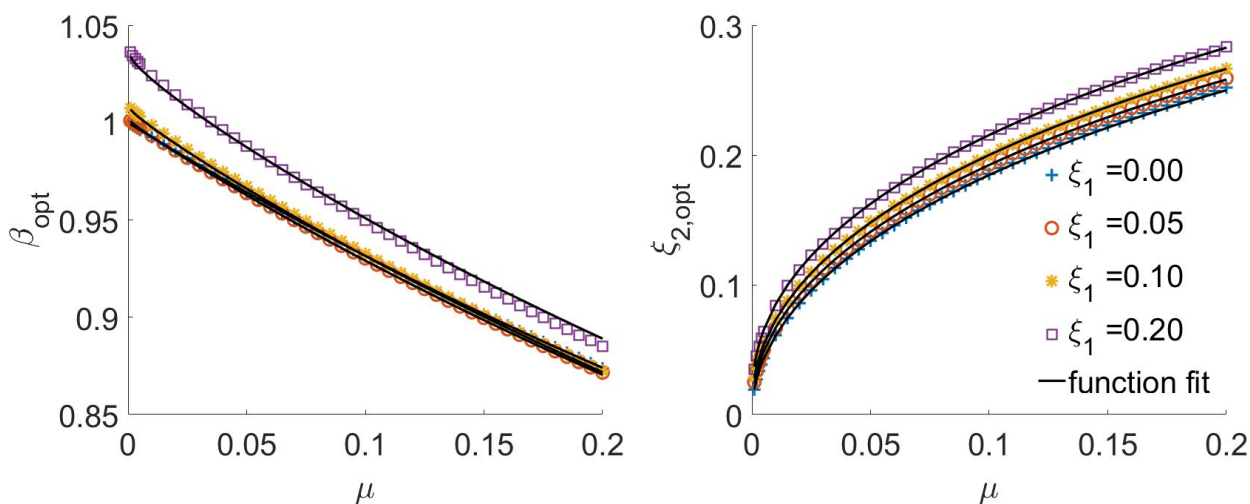

Figure 8. Optimum TMD parameters: TF No. 6.

complicated. As we can see in Figures 3-8, increase of $\xi_{1}$ leads to decrase of $\beta_{o p t}$ in the case of displacement FRFs (No. 1,3,5). In the case acceleration FRFs (No. $2,4,6)$, the increase of $\xi_{1}$ has an opposite effect. Sensitivity of optimum parameters to the damping ratio $\xi_{1}$ varies among FRFs. It is interesting to note that in the case of acceleration FRFs, the optimum tuning $\beta_{\text {opt }}$ may slightly rise above one, which is an unusual situation in TMD design.

\section{Conclusions}

The design formulas for $H_{\infty}$ optimization of TMD attached to a damped structure are presented in this paper. The formulas are appropriate for TMDs with the mass ratio $\mu$ between 0.001 and 0.2 , and for the damping ratio of the main structure between 0 and 
0.2. This is a sufficient range for most of TMDs localized in civil engineering structures and we consider the error of design parameters negligible in this range.

The formulas were optimized for six frequency response functions, which describe relative and absolute displacement or acceleration for both force and support excitation. It is necessary to chose a suitable FRF for TMD optimization regarding the purpose of TMD. For the purpose of a stress reduction of the main structure, FRFs which describe displacement should be used. On the other hand, in order to increase a comfort of people in high-rise buildings and pedestrian on footbridges, or to reduce the vibration of equipment localized in the main structure, the acceleration FRFs are an appropriate choice. We also find out that reduction of acceleration may lead to TMD tuned to a resonant or even slightly higher frequency.

An easy application is preserved. Only two simple formulas are presented, however, they can be used for all considered transfer functions with different parameters $a_{i}$ and $b_{i}$.

\section{ACKNOWLEDGEMENTS}

The authors gratefully acknowledge support from the Czech Technical University in Prague, project SGS20/038/OHK1/1T/11 Development and application of numerical algorithms for analysis and modelling in mechanics of structures and materials.

\section{REFERENCES}

[1] J. P. Den Hartog. Mechanical Vibrations. McGraw Hill, New York, 1934.

[2] J. Brock. A note on the damped vibration absorber. Journal of Applied Mechanics 68(A):284, 1946.

[3] T. Ioi, K. Ikeda. On the dynamic vibration damped absorber of the vibration systems. Bulletion of the JSME 21(151):64-71, 1978.

[4] SETRA. Footbridges: Assessment of vibrational behaviour of footbridges under pedestrian loading, 2006.

[5] S. E. Randall, D. M. Halsted, D. L. Taylor. Optimum vibration absorbers for linear damped systems. Journal of Mechanical Design 103:908-913, 1981.

[6] T. Asami, O. Nishihara, A. M. Baz. Analytical solutions to $H_{\infty}$ and $H_{2}$ optimization of dynamic vibration absorbers attached to damped linear systems. Journal of Vibration and Acoustics 124:284-295, 2002.

[7] I. M. Abubakar, B. J. M. Farid. Generalized den hartog tuned mass damper system for control of vibrations in structures. Earthquake Resistant Engineering Structures 104:185-193, 2009.

[8] FIB Bulletin 32: Guidelines for the design of footbridges, 2005.

[9] O. Nishihara, T. Asami. Closed-form exact solution to $\mathrm{h}$ infinity optimization of dynamic vibration absorber: Ii. development of an algebraic approach and its application to a standard problem. Proceedings of the SPIE, 3989, 2000. DOI:10.1117/12.384589. 